Article

\title{
Wireless Sensor Network (WSN) Configuration Method to Increase Node Energy Efficiency through Clustering and Location Information
}

\author{
Jinsoo Kim ${ }^{1}$, Donghwan Lee ${ }^{2}$, Jaejoon Hwang ${ }^{3} \mathbb{D}$, Sunghoon Hong ${ }^{1}$, Dongil Shin ${ }^{2}$ and Dongkyoo Shin ${ }^{2, *(D)}$ \\ 1 NetCom Systems Co., Ltd., \#1903, Daesung D-Polis Knowledge Industry Center A, Seobusaet-Gil 606, \\ Geumcheon-Gu, Seoul 08504, Korea; jskim@netcom.co.kr (J.K.); shhong@netcom.co.kr (S.H.) \\ 2 Department of Computer Engineering, Sejong University, Seoul 05006, Korea; dhwlee@sju.ac.kr (D.L.); \\ dshin@sejong.ac.kr (D.S.) \\ 3 Hanwha Systems, Seongnam 13524, Korea; jjhwang@naver.com \\ * Correspondence: shindk@sejong.ac.kr
}

check for updates

Citation: Kim, J.; Lee, D.; Hwang, J.; Hong, S.; Shin, D.; Shin, D. Wireless Sensor Network (WSN)

Configuration Method to Increase

Node Energy Efficiency through

Clustering and Location

Information. Symmetry 2021, 13, 390.

https://doi.org/10.3390/sym13030390

Academic Editors: Simona Halunga,

Octavian Fratu and Michel Planat

Received: 14 January 2021

Accepted: 22 February 2021

Published: 27 February 2021

Publisher's Note: MDPI stays neutral with regard to jurisdictional claims in published maps and institutional affiliations.

Copyright: (c) 2021 by the authors. Licensee MDPI, Basel, Switzerland. This article is an open access article distributed under the terms and conditions of the Creative Commons Attribution (CC BY) license (https:// creativecommons.org/licenses/by/ $4.0 /)$.

\begin{abstract}
Wireless Sensor Network (WSN) technology, for services that are difficult to access or which need to be continuously monitored regardless of location, needs further research and development due to an expansion of fields where it can be applied and due to increases in efficiency. In particular, in the field of defense, research on the latest IT technologies including sensor networks is being actively conducted as an alternative to the risky use of personnel in areas such as surveillance and surveillance reconnaissance. This paper experimented with analyzing the conditions necessary for increasing the energy efficiency of the nodes constituting a sensor network using a clustering routing technique and a location-based routing technique. The derived factors include a method for selecting a cluster head $(\mathrm{CH})$, a method for establishing a path from each channel to a base station (BS), and a method for transmitting collected data. We experimented with the derived factors and proposed a WSN configuration method that increases the energy efficiency of each node by applying optimal results and methods that were verified experimentally.
\end{abstract}

Keywords: Wireless Sensor Network (WSN); cluster head (CH); base station (BS); cluster-based routing protocol; location-based routing protocol

\section{Introduction}

Wireless Sensor Network (WSN) technology is suitable for applications that are difficult to access or require continuous monitoring due to geographical and physical factors. Recently, the importance of this technology has been highlighted due to the development of related technologies, an expansion of fields where it can be applied, and increases in efficiency. In particular, various sensor-related technologies based on low power are expanding WSN applications to private sectors, including large-scale manufacturing, defense areas aimed at monitoring, natural disaster response, and social safety nets [1-3]. An example of a sensor network use case in the defense field is an unmanned boundary system using Ubiquitous Sensor Network (USN) technology. In this regard, research is being conducted on the Surveillance and Reconnaissance Sensor Network (SRSN) to build an unmanned boundary system [1]. SRSN is a system that applies sensor network technology to transmit sensing information in real-time according to the function of a sensor in the field of surveillance reconnaissance.

The general physical configuration of the sensor network system applied to SRSN consists of a sensor node, base station (BS) or sinkhole, and a database/web server that collects necessary data, as shown in Figure 1. The sensor node performs the function of transmitting data to the BS after obtaining information such as magnetic, acoustic, vibration, and temperature readings [2,3]. 


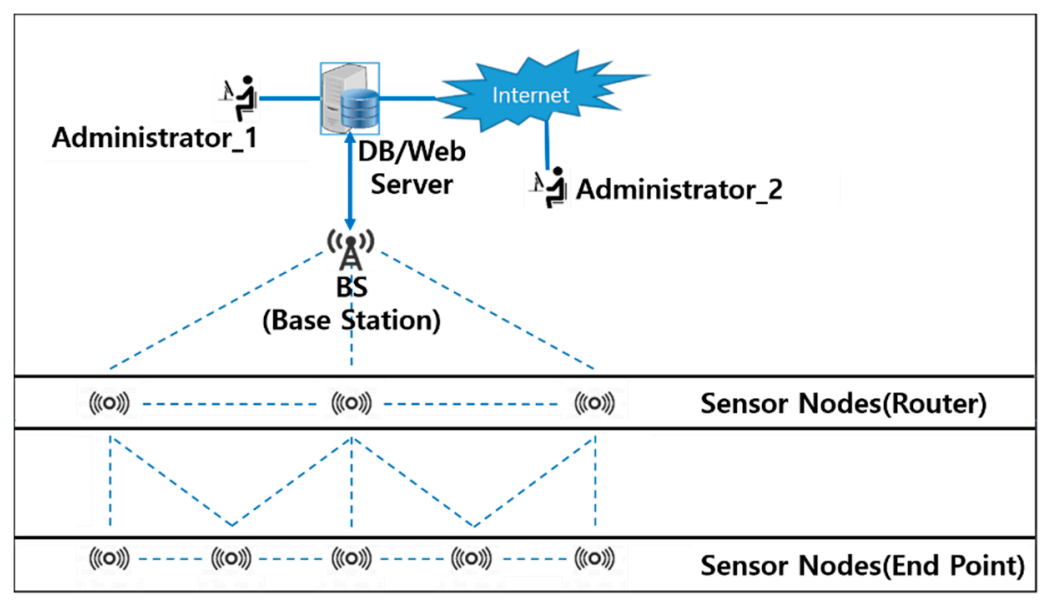

Figure 1. Wireless Sensor Network (WSN) physical structure.

The physical configuration of a typical WSN system is as follows: Sensor nodes that transmit data directly to the BS are classified as routers or gateways, and sensor nodes that transmit data to the routers or gateways are classified as end nodes. Sensor nodes corresponding to routers or gateways can reduce transmission and reception of meaningless data by applying a function of integrating the data of end nodes when designing and implementing a routing protocol. Additionally, the BS performs the function of receiving data from the sensor node and transmits it to the database server. Unlike normal sensor nodes, the BS uses constant power, thus it operates without being affected by energy consumption during data transmission and reception. The database/web server analyzes the data received from the BS and derives the desired results from the corresponding service, such as boundary and surveillance reconnaissance information, and performs the function of providing it to the administrator through the web server.

In this study, we propose a method to reduce energy consumption when transmitting/receiving data between sensor nodes and BSs by reflecting the physical structure of the WSN, thereby increasing the lifespan of the nodes and the service life of the entire sensor network.

The composition of this paper is as follows. Section 2 reviews related research that examines routing methods to reduce the energy consumption of sensor nodes. Section 3 proposes routing methods to reduce the energy consumption of sensor nodes. In Section 4, the performance of the proposed method is verified through simulation. Section 5 provides conclusions and future tasks.

\section{Related Work}

WSN studies can be divided into methods for improving the performance of the WSN system through performance improvement of the sensor node, which is a major component, and methods for improving performance by reducing energy consumption during data transmission/reception, such as through improvements in routing. In this paper, we proposed a method to improve the lifetime of a WSN through improvements in routing. Accordingly, we compare and analyze various proposed routing protocols for a WSN.

WSN routing protocols can be divided into flat-based protocols, hierarchy-based protocols, and location-based protocols. The features of each configuration method have various advantages and disadvantages.

\subsection{Flat-Based Protocol}

In a flat-based protocol, each sensor node performs the same role and function and also includes the ability to collaborate with neighboring nodes to collect data. Data transmission to the BS exchanges control information with neighboring nodes to establish a transmission path and transmits the data through the established path. Typical protocols using a flat- 
based protocol include Sensor Protocols for Information via Negotiation (SPIN) [3,4] and Direct Diffusion [4-6].

SPIN adopts the concept of metadata and uses a negotiation technique that ensures that only useful data is transmitted when exchanging data between neighboring sensor nodes. This minimizes implosion and overlapping problems that occur in a flat-based protocol when flooding. SPIN has a drawback in that an individual sensor node needs only information about its immediate neighbor node, and if a very simple protocol or destination exists far away and a sensor node in the middle does not request data transmission, it may fail to transmit data to its destination. Figure 2 shows the process of transmitting to neighboring nodes using the above three types of messages when sensor node A collects new data in SPIN.

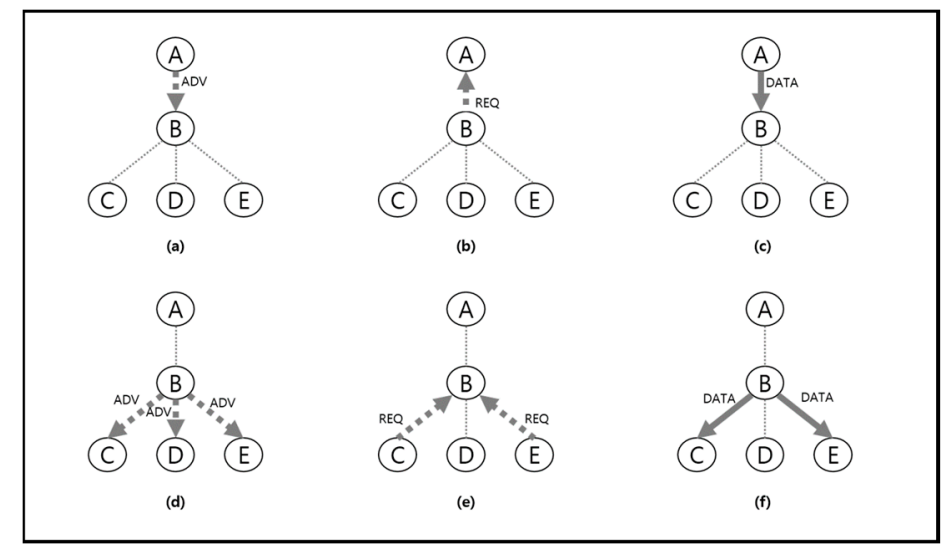

Figure 2. Sensor Protocols for Information via Negotiation (SPIN) data transmission process. Node A starts by advertising its data to node B (a). Node B responds by sending a request to node A (b). After receiving the requested data (c), node $B$ then sends out advertisements to its neighbors (d), who in turn send requests back to $B(\mathbf{e}, \mathbf{f})$.

Direct Diffusion is a data-centric routing protocol that propagates requests using interest inquiries from the BS and sends data through reports from sensor nodes with events matching the interest. In this method, the path from the sensor node to the BS is established by applying an enhancement function of the path generated in the opposite direction to the gradient, which is the propagation path of interest. Therefore, if the requested content of the BS is continuous, it can be used properly because data is transmitted to the route set through route reinforcement. However, if the request is a one-time event, it is difficult to properly apply because it requires continuous interest inquiry and route reinforcement. Figure 3 shows the interest propagation process in the BS and the process of reporting an event to the BS from a sensor node that detects an event matching the interest.

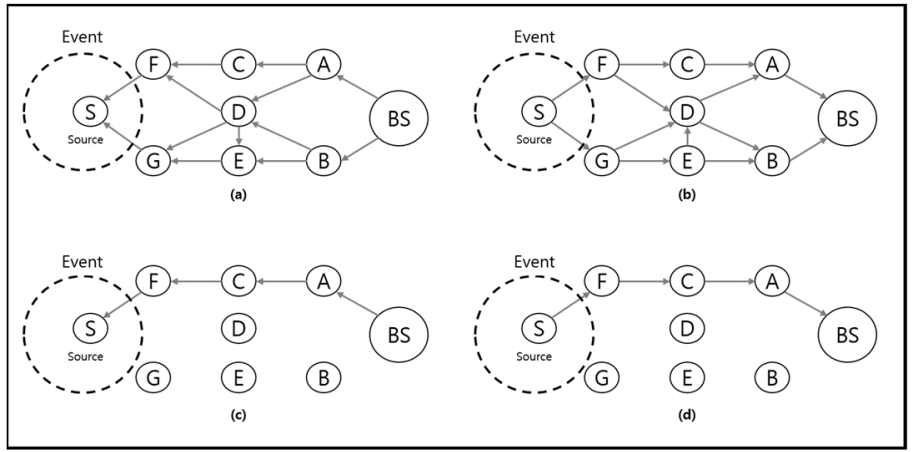

Figure 3. Direct Diffusion data transmission process. (a) Interest propagation. (b) Gradient establishment. (c) Send probe data. (d) Reinforce path. 


\subsection{Hierarchy-Based Protocol}

In a hierarchy-based protocol, after dividing the entire network into several groups called clusters, a cluster head $(\mathrm{CH})$ representing an individual cluster is selected, and then the $\mathrm{CH}$ transmits data on behalf of the cluster. The $\mathrm{CH}$ collects data and receives data collected by general sensor nodes in the cluster, and then performs data aggregation and transmits the data to the BS. Therefore, $\mathrm{CH}$ may have problems in terms of energy consumption compared to normal sensor nodes, and resolution requires the ability to change the $\mathrm{CH}$ by taking into account the selection of $\mathrm{CH}$, the duration of the $\mathrm{CH}$ role, etc., as well as the derivation of the required number of clusters across the entire network and the scope of clusters. Typical examples of hierarchy-based protocols are Low-Energy Adaptive Clustering Hierarchy (LEACH) [7] and Energy-Efficient Uneven Clustering (EEUC) [8].

LEACH is the beginning of a hierarchy-based protocol for WSN, and clusters the entire network to create groups and then selects the $\mathrm{CH}$ for each cluster. At this time, the $\mathrm{CH}$ manages all sensor nodes in the cluster, and the data transmitted by the sensor nodes are integrated and transmitted to the BS. Therefore, $\mathrm{CH}$ consumes more energy than a general sensor node. To address this, a separate $\mathrm{CH}$ is selected for each round. When selecting a $\mathrm{CH}$ in $\mathrm{LEACH}$, all sensor nodes in the cluster are eligible to be equally selected as $\mathrm{CH}$, which causes all sensor nodes to consume energy evenly. However, a limitation of LEACH is that it does not reflect energy consumption according to the physical location of the sensor node when selecting $\mathrm{CH}$.

Based on the distance between the BS and the sensor nodes, EEUC configures a cluster with a smaller range of clusters located closer to the BS than other clusters. This is because the $\mathrm{CH}$ in the cluster close to the BS performs a relay function of data transmitted from the other $\mathrm{CH}$ to the BS, so energy consumption is large. However, as shown in Figure 4, EEUC operates smoothly when sensor nodes close to the BS are evenly distributed. There is a disadvantage in this approach in that the energy of the sensor nodes adjacent to the BS is rapidly consumed.

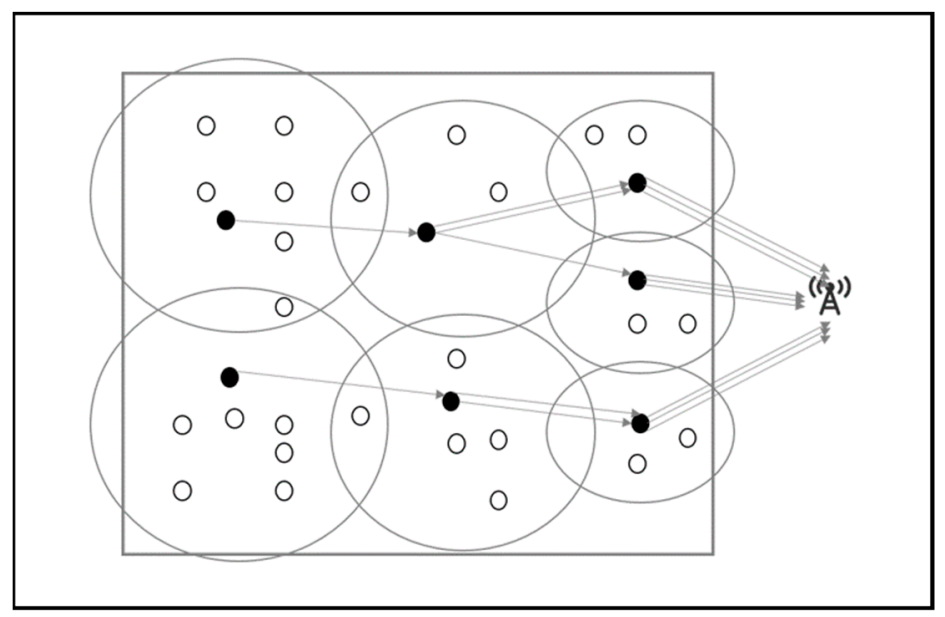

Figure 4. Energy-Efficient Uneven Clustering (EEUC) operation process.

\subsection{Location-Based Protocol}

A location-based protocol uses the location information of the sensor node and BS to set the transmission path of the collected data. Therefore, a location-based protocol does not require separate energy consumption for path setting or maintenance. Geographic and Energy-Aware Routing (GEAR) [9] is a representative example that can minimize bandwidth consumption.

GEAR is divided into a step that transmits a packet from a BS to a target region and another step to propagate a packet within a target region. When transmitting packets from the BS to the target area, gradual routing is used so that neighboring sensor nodes use energy evenly by applying the concept of learning cost and prediction cost. In the 
packet propagation stage within the region, a recursive transmission method using location information is applied instead of flooding, which consumes much energy. The recursive transmission technique is a method of dividing a target region into sub-regions and then copying and propagating packets to each sub-region. However, when the density of the sensor node in the target area is low and the communication range of the sensor node does not reach the entire sub-area, packet propagation is performed inside the area using limited flooding, which limits the hop count of packets to 1 or 2 .

\section{Suggested Techniques}

\subsection{WSN Operating Process}

The proposed WSN routing method simultaneously applies a cluster-based protocol and location-based protocol. Figure 5 shows the detailed configuration and operation process of the WSN. Cluster selection can be preset in a grid pattern due to the introduction of a location-based protocol, and each node collects the cluster information based on the acquired location information and then sets the $\mathrm{CH}$ to transmit the collected information to the $\mathrm{CH}$ of the cluster. $\mathrm{CH}$ integrates this and transmits it to $\mathrm{BS}$, and when transmitting data from $\mathrm{CH}$ to BS, a Shortest Path First (SPF) algorithm is applied to minimize energy consumption during long-distance transmission.

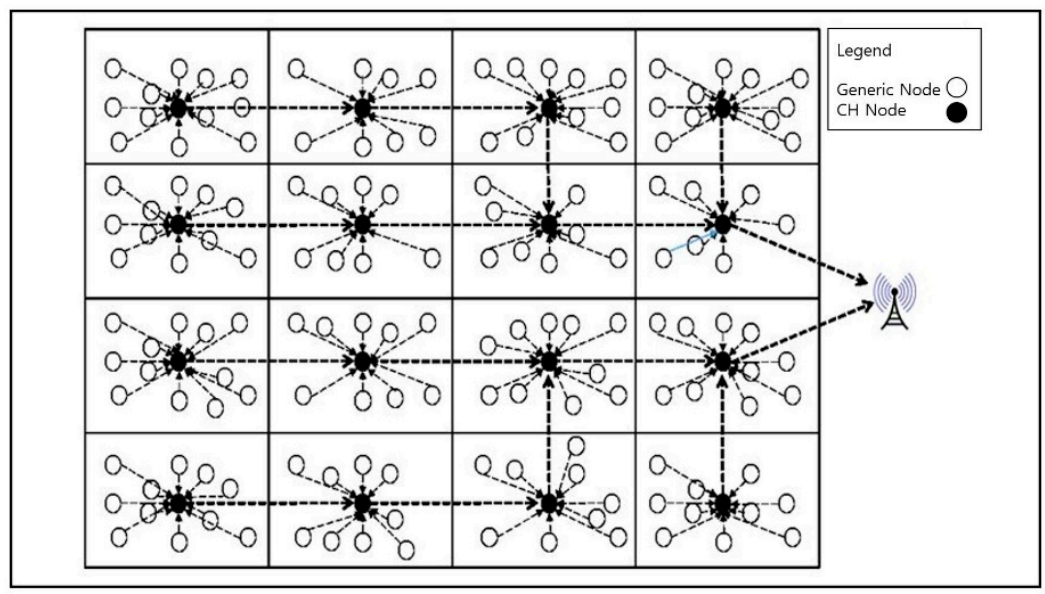

Figure 5. WSN operating process.

\subsection{Performance Improvement Considerations for WSN Configuration}

In this study, we pre-setup clusters based on location information and obtain cluster information to which the nodes belong to derive factors that affect the performance of WSN, such as shown in Table 1, which include the following considerations.

Table 1. WSN performance improvement considerations.

\begin{tabular}{cll}
\hline Sortation & \multicolumn{1}{c}{ Considerations } \\
\hline CH Selection & - & $\begin{array}{l}\text { Residual energy of sensor node } \\
\text { Position of sensor node (distance from the center } \\
\text { of cluster) }\end{array}$ \\
\hline Choosing a route between CH and BS & - & $\begin{array}{l}\text { Energy consumption during direct communication } \\
\text { between } \mathrm{CH} \text { and BS by way of } \mathrm{CH}\end{array}$ \\
\hline
\end{tabular}

\subsection{Suggested Routing Protocol}

The proposed routing protocol suggests the way to minimize energy consumption in an environment where each node acquires physical location and cluster information in advance. In addition, through this method, energy consumption is evenly distributed 
among nodes to improve network life and ensure smooth operation. The proposed protocol is divided into a $\mathrm{CH}$ selection step, a channel selection step between $\mathrm{CH}$ and $\mathrm{BS}$, and a collection data transmission step.

\subsection{1. $\mathrm{CH}$ Selection}

The proposed protocol works for each round like LEACH. CH selection for each cluster is selected in consideration of the residual energy of each sensor node and the position of the sensor node derived from the WSN performance improvement considerations of Table 1. Each cluster selects a $\mathrm{CH}$ that transmits data to BS every round, and a score function is introduced for $\mathrm{CH}$ selection. The conditions of the node to be selected as $\mathrm{CH}$ are as follows:

1. Nodes with high residual energy.

2. Nodes close to the center of the cluster. It is possible to calculate the center of the cluster and the distance of the sensor nodes by using the Global Positioning System (GPS) installed by each sensor node.

The reason for introducing the weight is to determine which of the above conditions should be weighted. The following score function is introduced for this purpose:

$$
\operatorname{Score}\left(C H_{i}\right)=\alpha E_{i}+(1-\alpha) \frac{1}{D_{i}^{2}}
$$

In Equation (1), $E_{i}$ is the amount of remaining energy of sensor node $i$ in a cluster, $D_{i}$ is the distance between sensor node $i$ and the center of the cluster, and $\alpha(0 \leq \alpha \leq 1)$ is the weight. By applying Equation (1), the node with the highest score in each cluster is selected as $\mathrm{CH}$. When selecting the $\mathrm{CH}$ node, the selection of the weight $(\alpha)$ can affect the performance of the proposed routing protocol, so the optimal weight was selected by performing the following simulation.

Figure 6 shows that the results in a round with 100 nodes are down when weight $(\alpha)$ is 0.8 . If 100 nodes go down in the 522nd round, it provides optimal results. Based on this result, this study applied 0.8 to weight $(\alpha)$.

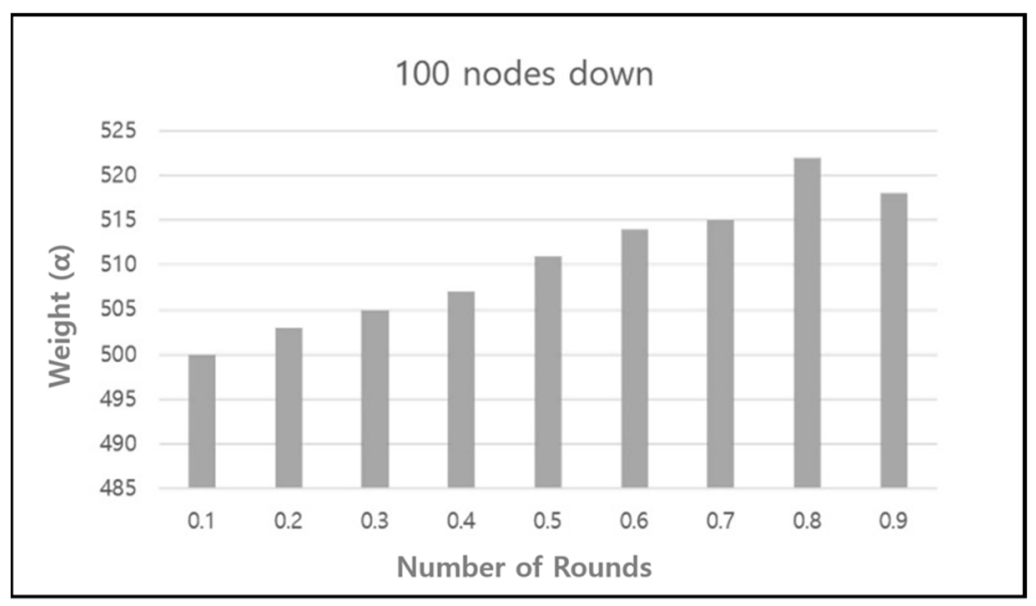

Figure 6. Round when 100 nodes by weight are down.

\subsubsection{Route Selection between $\mathrm{CH}$ and BS}

After the $\mathrm{CH}$ is selected, a path setting step is required to transmit data to the BS. Transmitting data directly from $\mathrm{CH}$ to BS consumes a considerable amount of energy due to the relatively large transmission distance. To solve this problem, the shortest transmission path between $\mathrm{CH}$ and BS is selected by applying a Dijkstra algorithm [10]. To this end, the operation process is as follows: 
1. Nodes selected as $\mathrm{CH}$ transmit node location information to BS.

2. BS calculates the shortest transmission path between BSs in individual CHs by using the location information of the aggregated $\mathrm{CH}$ nodes.

3. BS transmits the calculated shortest path (next hop of individual $\mathrm{CH}$ ) to the corresponding $\mathrm{CH}$.

\subsubsection{Collection Data Transmission}

The nodes belonging to the cluster transmit the collected data to the $\mathrm{CH}$ of the corresponding cluster, and the $\mathrm{CH}$ performs the process of collecting and integrating the received data. According to Reference [11], more than $50 \%$ of the total energy consumed by WSN occurs during data transmission. Therefore, the process of integrating aggregated data to reduce the amount of data transmitted from $\mathrm{CH}$ to BS is recognized as an important function. The $\mathrm{CH}$ transmits the integrated data to the BS, and the BS collects the data received from each $\mathrm{CH}$ and sends it to the database/web server to perform the service.

\section{Performance Evaluation}

To evaluate the performance of the proposed routing protocol, we utilized LEACH, a representative routing protocol, and did performance analysis. The performance evaluation criteria considered the number of surviving nodes and the amount of energy consumed by the surviving nodes after each round of transmission.

\subsection{Performance Evaluation Environment}

The simulation was conducted using $C$ language, and the WSN range was set to $200 \times 200$, and 16 clusters with a range of $50 \times 50$ were applied. The sensor node randomly placed 200 nodes, and the BS determined a fixed location to perform performance evaluation. In addition, to reduce the variation in data values caused by random node positioning, the performance evaluation results averaged the values performed in 100 topologies. Table 2 shows the values of the main variables used in the performance evaluation.

Table 2. Performance evaluation environment variables.

\begin{tabular}{ll}
\hline \multicolumn{1}{c}{ Environment Variable } & \multicolumn{1}{c}{ Value } \\
\hline BS (Base Station) & $(210,210)$ \\
\hline Number of Nodes & 200 \\
\hline Initial energy/node & $20 \mathrm{~J}$ \\
\hline$E_{\text {elec }}$ & $50 \mathrm{~nJ} / \mathrm{bit}$ \\
\hline$E_{f s}$ & $7 \mathrm{~nJ} / \mathrm{bit} / \mathrm{m}^{2}$ \\
\hline$E_{a m p}$ & $100 \mathrm{pJ} / \mathrm{bit} / \mathrm{m}^{2}$ \\
\hline$E_{m p}$ & $0.0013 \mathrm{pJ} / \mathrm{bit} / \mathrm{m}^{2}$ \\
\hline Control Data Size & $10 \mathrm{Byte}(80 \mathrm{bit})$ \\
\hline Packet Size & 250 Byte $(2000 \mathrm{bit})$ \\
\hline
\end{tabular}

\subsection{Protocol Performance Evaluation Result and Analysis}

To evaluate the performance of the proposed protocol, the number of surviving nodes per round was compared and analyzed for direct communication, LEACH protocol, and proposed technique. As shown in Figure 7, after performing 200 rounds, 39 nodes, 20\% of all nodes, survive. In contrast, in the proposed protocol and LEACH, more than 95\% of all nodes survive after 200 rounds. After 400 rounds, the proposed protocol shows that more than $90 \%$ of the 181 nodes survived, while for LEACH, $47 \%$ of the 94 nodes survived, indicating that the proposed protocol survived by $43 \%$ or more compared to LEACH. This is because the proposed protocol up to 400 rounds uses energy more evenly and efficiently at the sensor node compared to LEACH. Surviving nodes are the same number in the 
proposed protocol and LEACH at 700 rounds, where both measures cannot be significant because only $3 \%$ of the nodes survive and cannot perform the services of WSN.

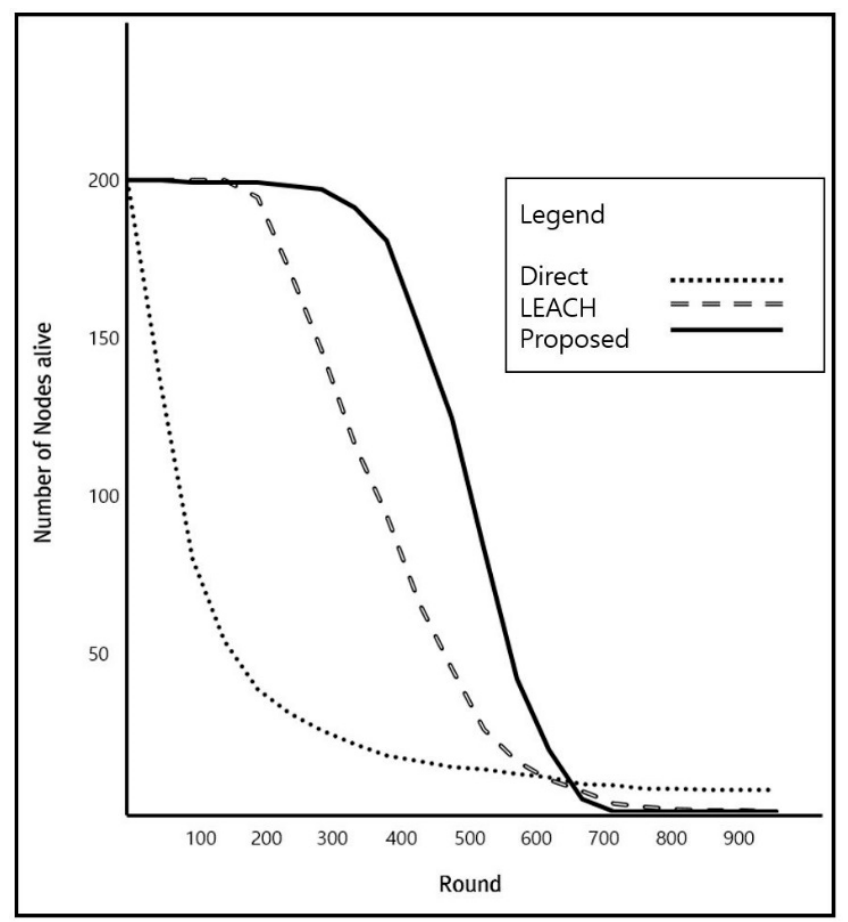

Figure 7. Survival nodes per round.

The singularity is that after 700 rounds, the direct communication method survives with more nodes than LEACH or the proposed method. The reason is that as shown in Figure 8, a few sensor nodes located close to the BS do not consume much energy due to the relatively small distance when transmitting data to the BS. However, these are only $4.5 \%$ (9) of the surviving sensor nodes, and in this situation, the WSN service cannot be smoothly performed.

\begin{tabular}{|c|c|c|c|}
\hline $\begin{array}{llll}0 & 0 & 0 & 0 \\
0 & 0 & 0 & 0 \\
0 & 0 & 0 & 0 \\
0 & 0\end{array}$ & $\begin{array}{llll}0 & 0 & 0 & 0 \\
0 & 0 & 0 & 0 \\
0 & 0 & 0 & 0 \\
0 & 0\end{array}$ & $\begin{array}{lllll}0 & & 0 & 0 & 0 \\
0 & 0 & 0 & 0 \\
0 & 0 & 0 & 0 & 0\end{array}$ & $\begin{array}{llll}0 & 0 & 0 \\
0 & 0 & 0 & 0 \\
0 & 0 & 0 & 0 \\
0 & 0 & 0\end{array}$ \\
\hline $\begin{array}{llll}0 & 0 & 0 \\
0 & 0 & 0 \\
0 & 0 & 0 & 0 \\
0 & 0 & 0\end{array}$ & $\begin{array}{llll} & 0 & 0 & \\
0 & 0 & 0 \\
0 & 0 & 0 & 0 \\
0 & 0\end{array}$ & $\begin{array}{|lllll|}0 & & 0 & 0 & \\
0 & 0 & & 0 & 0 \\
0 & 0 & & 0 \\
\end{array}$ & $\begin{array}{llll}0 & 0 & \\
0 & 0 & 0 & 0 \\
0 & 0 & 0\end{array}$ \\
\hline $\begin{array}{llll}0 & 0 & 0 & 0 \\
0 & 0 & 0 & 0 \\
0 & 0 & 0 & 0\end{array}$ & $\begin{array}{llll}0 & 0 & 0 & 0 \\
0 & 0 & 0 & 0 \\
0 & 0 & 0 & 0\end{array}$ & $\begin{array}{lllll}0 & & 0 & 0 & 0 \\
0 & 0 & 0 & 0 \\
0 & 0 & 0 & 0 \\
0 & 0 & 0\end{array}$ & $\begin{array}{llll}0 & 0 & 0 & 0 \\
0 & 0 & 0 & 0 \\
0 & 0 & 0 & 0\end{array}$ \\
\hline $\begin{array}{llll}0 & 0 & 0 \\
0 & 0 & 0 & 0 \\
0 & 0 & 0 & 0 \\
0 & 0\end{array}$ & $\begin{array}{llll}0 & 0 & \\
0 & 0 & 0 \\
0 & 0 & 0 & 0 \\
0 & 0\end{array}$ & $\begin{array}{lllll}0 & & 0 & 0 \\
0 & 0 & 0 & \\
0 & 0 & 0 & 0 \\
0 & 0 & 0\end{array}$ & $\begin{array}{llll}0 & 0 & 0 \\
0 & 0 & 0 & 0 \\
0 & 0 & 0\end{array}$ \\
\hline
\end{tabular}

Figure 8. Distribution of surviving nodes among direct communication nodes after 700 rounds.

Figure 9 shows the average energy consumption of each node per round. After 50 rounds, the proposed protocol consumes $30-40 \%$ less energy than the average LEACH per node, and the trend continues, and after 300 rounds, the average consumes more than $30 \%$ less energy per node than LEACH. This means that the sensor nodes in the proposed protocol use energy more uniformly and efficiently than LEACH. After 700 rounds, the average energy consumption per node shows near-similar energy consumption in both the proposed protocol and LEACH. At this point, both protocols are alive with only sensor 
nodes of less than $5 \%$ and therefore WSN's services cannot be performed and cannot be considered. In addition, the proposed protocol consumes $70 \%$ to $80 \%$ less energy than direct communication per node up to 200 rounds, and the difference gradually becomes smaller. This is because in direct communication, most nodes consume energy in the first 200 rounds, and more than $80 \%$ of the nodes afterwards do not work properly.

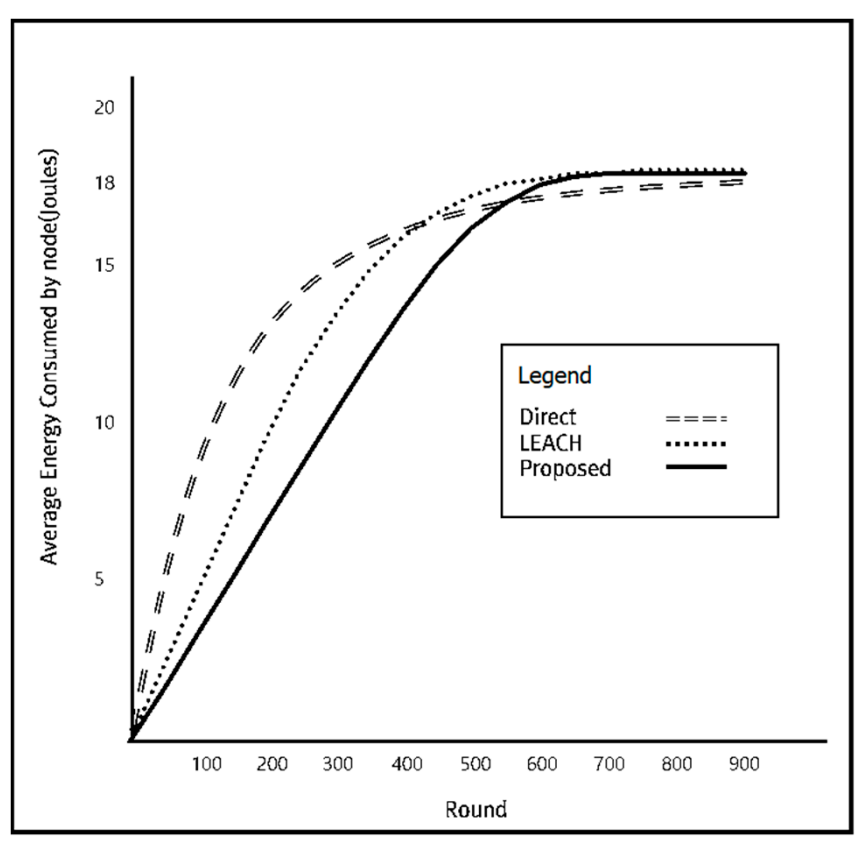

Figure 9. Average energy consumption per node per round.

Figure 10 shows the residual energy distribution of all sensor nodes after 400 rounds of communication by applying the proposed protocol. When a cluster is located closer to the BS, the residual energy of the sensor nodes existing in the cluster decreases. In the proposed protocol, $\mathrm{CHs}$ located at a distance from the $\mathrm{BS}$ transmit data via a $\mathrm{CH}$ adjacent to the BS without transmitting data directly to the BS to reduce energy consumption due to long-distance data transmission. That is, nodes in the cluster adjacent to the BS consume a large amount of energy for relay transmission of data transmitted from the other different $\mathrm{CHs}$ to the BS.

\begin{tabular}{|c|c|c|c|c|}
\hline $\begin{array}{lllll}0 & 0 & 0 & 0 \\
0 & 0 & 0 & 0 & 0 \\
0 & 0 & 0 & 0 \\
0\end{array}$ & $\begin{array}{llll}0 & 0 & 0 & 0 \\
0 & 0 & 0 & 0 \\
0 & 0 & 0 \\
0 & 0 & 0 \\
0\end{array}$ & $\begin{array}{ccccc}0 & 0 & 0 & 0 \\
0 & 0 & 0 & 0 & 0 \\
0 & 0 & 0 & 0 \\
0\end{array}$ & $\begin{array}{cccc}0 & 0 & 0 & 0 \\
0 & 0 & 0 & 0 \\
0 & 0 & 0 & 0 \\
0 & 0 & 0\end{array}$ & \\
\hline $\begin{array}{lllll}0 & 0 & 0 & \\
0 & 0 & 0 & 0 \\
0 & 0 & 0 & 0\end{array}$ & $\begin{array}{lllll}0 & & 0 & & \\
0 & 0 & 0 & 0 \\
0 & 0 & 0 & 0 \\
0 & 0 & 0\end{array}$ & $\begin{array}{lllll}0 & 0 & 0 & \\
0 & 0 & 0 & 0 \\
0 & 0 & 0 & 0\end{array}$ & $\begin{array}{llll} & & 0 & \\
0 & 0 & 0 & 0 \\
0 & 0 & 0 & 0 \\
0 & 0 & 0\end{array}$ & \\
\hline $\begin{array}{lllll}0 & 0 & 0 & 0 \\
0 & 0 & 0 & 0 \\
0 & 0 & 0 & 0 \\
0 & 0 & 0\end{array}$ & $\begin{array}{lllll}0 & & 0 & 0 & 0 \\
0 & 0 & 0 & 0 \\
0 & 0 & 0 & 0 \\
0 & 0 & 0\end{array}$ & $\begin{array}{lllll}0 & & 0 & 0 & 0 \\
0 & 0 & 0 & 0 \\
0 & 0 & 0 & 0\end{array}$ & $\begin{array}{llll}0 & 0 & 0 & 0 \\
0 & 0 & 0 & 0 \\
0 & 0 & 0 & 0\end{array}$ & \\
\hline $\begin{array}{llll}0 & & 0 & \\
0 & 0 & 0 & 0 \\
0 & 0 & 0 & 0 \\
0 & 0 & 0\end{array}$ & $\begin{array}{lllll}0 & & 0 & 0 \\
0 & 0 & 0 & 0 & 0 \\
0 & 0 & 0 & 0\end{array}$ & $\begin{array}{lllll}0 & & 0 & 0 \\
0 & 0 & 0 & 0 \\
0 & 0 & 0 & 0 \\
0 & 0 & 0\end{array}$ & $\begin{array}{llll}0 & 0 & 0 \\
0 & 0 & 0 & 0 \\
0 & 0 & 0\end{array}$ & \\
\hline
\end{tabular}

Figure 10. Residual energy distribution of sensor nodes after 400 rounds (proposed protocol).

\section{Conclusions}

In this paper, we proposed a method to extend the service life of WSN by improving the uniform energy use in sensor nodes by simultaneously applying the cluster-based 
protocol and the location-based protocol. The performance of the proposed protocol was evaluated and analyzed through simulation, and the proposed protocol showed improved results in terms of the number of surviving nodes per round and the average energy consumption per node per round compared to the comparative protocol, LEACH. Compared to LEACH, which sequentially selects $\mathrm{CH}$, the proposed protocol selects $\mathrm{CH}$ based on the sensor node's location information and residual energy, so that the energy of each sensor node can be used more evenly and efficiently. In addition, the simulation shows that $\mathrm{LEACH}$ sends data directly from all $\mathrm{CH}$ to $\mathrm{BS}$, while the proposed protocol reduces energy consumption by allowing $\mathrm{CH}$ to pass if there is a $\mathrm{CH}$ in the middle of the path to reduce energy consumption when transmitting data from $\mathrm{CH}$ to BS.

However, looking at the energy consumption distribution of each node in the proposed protocol, as the distance from the base station increases, the energy consumption of the sensor node adjacent to the base station increases and the energy consumption decreases. This is a phenomenon that occurs when a channel of a clusters adjacent to the base station continuously relays data transmitted by another channel to the base station. To solve this problem, it is necessary to study how to set the optimal number of clusters and cluster range by using the total number of sensor nodes of the WSN and the location information of each sensor node. In addition, the BS's location-based approach [12] can overcome the limitations revealed by the Restriction Protocol. In future research, we will continue to study two methods, how to determine the number of clusters to be set and how to transmit access depending on the location of the base station.

Author Contributions: J.K. and D.S. (Dongkyoo Shin) proposed the WSN configuration method detection and identified papers for the review; S.H., D.S. (Dongil Shin) and J.H. reviewed the papers, gathering information; J.K. and D.L. wrote the main manuscript text; D.S. (Dongkyoo Shin) reviewed the manuscript. All authors have read and agreed to the published version of the manuscript.

Funding: This work was supported by the Defense Acquisition Program Administration and Agency for Defense Development under the contract UD190016ED.

Institutional Review Board Statement: Not applicable.

Informed Consent Statement: Not applicable.

Data Availability Statement: Not applicable.

Conflicts of Interest: The authors declare no conflict of interest.

\section{References}

1. Ramson, S.R.J.; Moni, D.J. Applications of wireless sensor networks-A survey. In Proceedings of the 2017 International Conference on Innovations in Electrical, Electronics, Instrumentation and Media Technology (ICEEIMT), Coimbatore, India, 3-4 February 2017; pp. 325-329.

2. Xu, X.; Fallahi, N.; Yang, H. Efficient CUF-based FEM analysis of thin-wall structures with Lagrange polynomial expansion. Mech. Adv. Mater. Struct. 2020, 1-22. [CrossRef]

3. Shivalingagowda, C.; Jayasree, P.V.Y.; Sah, D.K. Efficient Energy and Position Aware Routing Protocol for Wireless Sensor Networks. KSII Trans. Internet Inf. Syst. 2020, 14, 1929-1950.

4. Raman, C.J.; Javid Ali, L.; Gobalakrishnan, N.; Pradeep, K. An Overview of the Routing Techniques Employed in Wireless Sensor Network. In Proceedings of the 2020 International Conference on Communication and Signal Processing (ICCSP), Chennai, India, 28-30 July 2020; pp. 332-336.

5. Yu, X.; Li, F.; Li, T.; Wu, N.; Wang, H.; Zhou, H. Trust-based secure directed diffusion routing protocol in WSN. J. Ambient Intell. Humaniz. Comput. 2020, 1-13. [CrossRef]

6. Intanagonwiwat, C.; Govindan, R.; Estin, D.; Heidemann, J.; Silva, F. Directed Diffusion for WSN. IEEE/ACM 2003, 11, 2-16. [CrossRef]

7. Daanoune, I.; Abdennaceur, B.; Ballouk, A. A comprehensive survey on LEACH-based clustering routing protocols in Wireless Sensor Networks. Ad Hoc Netw. 2021, 114, 102499. [CrossRef]

8. Ullah, Z. A Survey on Hybrid, Energy Efficient and Distributed (HEED) Based Energy Efficient Clustering Protocols for Wireless Sensor Networks. Wirel. Pers. Commun. 2020, 124, 2685-2713. [CrossRef]

9. Zagrouba, R.; Kardi, A. Comparative Study of Energy Efficient Routing Techniques in Wireless Sensor Networks. Information 2021, 12, 42. [CrossRef] 
10. Barbehenn, M. A note on the complexity of Dijkstra's algorithm for graphs with weighted vertices. IEEE Trans. Comput. 1998, 47, 263. [CrossRef]

11. Halgamuge, M.N.; Nukerman, M.; Ramamohanarao, K. An estimation of sensor energy consumption. Prog. Electromagn. Res. B 2009, 12, 259-295. [CrossRef]

12. Roy, S.; Mazumdar, N.; Pamula, R. An optimal mobile sink sojourn location discovery approach for the energy-constrained and delay-sensitive wireless sensor network. J. Ambient Intell. Humaniz. Comput. 2021, 1-28. [CrossRef] 